\title{
Jardines luminiscentes en las salinas de las Islas Canarias
}

\section{Lumiscent gardens in the saltworks of the Canary Islands}

\author{
Víctor Manuel Cabrera García ${ }^{(*)}$
}

\section{RESUMEN}

Recuperar el patrimonio salinero canario en desuso mediante la incorporación de nuevos usos que sean compatibles con las salinas supone un reto importante para nuestra sociedad. Ante el manifiesto estado de abandono que tienen actualmente las salinas de las Islas Canarias se propone revitalizarlas desde la óptica del paisaje creando jardines luminiscentes en aquellas salinas que han quedado desnaturalizadas en la trama urbana atendiendo al fenómeno natural de la bioluminiscencia. Este novedoso concepto de jardines podría convertirse en nuevos polos de atracción para el turismo cultural, lo que permitiría generar nuevas experiencias sensoriales que aportan valores añadidos respecto a las diversas rutas culturales y patrimoniales que se realizan en unos paisajes marinos tan singulares como son las salinas.

Palabras clave: Salinas; revitalizar; patrimonio; jardines; luminiscencia

\section{ABSTRACT}

Recover the unused salt heritage of Canary Island trough new uses incorporation that are compatible with the saltworks, it is an important challenge for our society. Given the manifest of the abandonment state that have now a days the salttworks of Canary Islands, it is suggested revitalize them from the perspective of the landscape, creating luminescent gardens in those saltworks that have been denatured in the urban layout and attending to the natural phenomenon of bioluminescence. This original concept of luminescent gardens could become in new cultural centres of tourist attraction that it will be allowed to generate new sensory experiences that contribute added values respect to the diverse cultural and heritage routes that take place in marine landscapes as unique as the saltworks.

Keywords: Saltworks; revitalize; heritage; gardens; luminiscence

(*) Dr. arquitecto. Docente e investigador del Departamento de Construcción Arquitectónica. Escuela de Arquitectura. Universidad de Las Palmas de Gran Canaria (España).

Persona de contacto/Corresponding author: victormanuel.cabrera@ulpgc.es (V. M. Cabrera García)

ORCID: http://orcid.org/oooo-0002-8203-3169 (V. M. Cabrera García)

Cómo citar este artículo/Citation: Víctor Manuel Cabrera García (2021). Jardines luminiscentes en las salinas de las Islas Canarias. Informes de la Construcción, 73(563): e410. https://doi.org/10.3989/ic.81028

Copyright: (C) 2021 CSIC. Este es un artículo de acceso abierto distribuido bajo los términos de la licencia de uso y distribución Creative Commons Reconocimiento 4.0 Internacional (CC BY 4.0). 


\section{INTRODUCCIÓN}

El paisaje salinero es una realidad compleja y dinámica, compuesta por elementos naturales y culturales, materiales e inmateriales, tangibles e intangibles, consecuencia de los procesos de producción y comercialización de la sal sobre el territorio a lo largo de los siglos. Las salinas son, por tanto, paisajes culturales cuya definición viene recogida, entre otros documentos, en el Plan Nacional de Paisaje Cultural como el "resultado de la interacción en el tiempo de las personas y el medio natural, cuya expresión es un territorio percibido y valorado por sus cualidades culturales, producto de un proceso y soporte de la identidad de una comunidad" (1)

El Convenio Europeo del Paisaje del año 2000 define a las salinas como lugares de características únicas resultado de un proceso artesanal de producción y comercialización de la sal, que se obtiene a partir de una serie de construcciones situadas tanto en los territorios costeros como en los de interior. Estos espacios tienen valores naturales, medioambientales, culturales, patrimoniales y sociales resultado de la acción e interacción de factores naturales y humanos en el territorio. (2)

En la actualidad la mayoría de las salinas de Canarias están en un estado avanzado de ruinas y de abandono debido a la falta de actividad. Si no se realizan labores de mantenimiento, protección y puesta en uso de estas instalaciones en los próximos años consentiremos la pérdida, tanto para la sociedad actual como para la sociedad futura, de un valioso patrimonio cultural y paisajístico.

En este sentido, la Ley 4/1999 sobre el Patrimonio Histórico de Canarias que posteriormente es modificada por la Ley 11/2002 del 21 de noviembre cita lo siguiente "Se declaran bienes de interés cultural del patrimonio histórico canario aquellos bienes que ostenten notorios valores históricos, arquitectónicos, artísticos, arqueológicos, etnográficos o paleontológicos o que constituyan testimonios singulares de la cultura canaria”. (3)

La UNESCO define a las salinas como espacios a proteger por su alto valor ecológico, no solo en referencia a la obtención artesanal de la sal, sino que son espacios que por sí mismos poseen una gran riqueza ecológica en cuanto a su flora, fauna y las comunidades que forman. Las especies vegetales más integradas en el ambiente acuático son las diminutas algas microscópicas que viven flotando en el seno del agua y están sujetas a sus movimientos y cambios de composición. Estas algas dependen por completo del agua, constituyen el denominado fitoplacton y son la base para la alimentación de todos los animales que viven en esta comunidad.

El deterioro existente en las salinas supone una gran pérdida de cualidades de un paisaje singular y una gran pérdida patrimonial, tanto de la estructura de muretes, cocederos y tajos como de los antiguos molinos de viento tradicionales, hitos del paisaje que hoy aparecen en estado de abandono. El patrimonio intangible asociado a estas infraestructuras configura también un bien cultural de primer orden cuya continuidad depende de que la actividad y de su cultura material (patrimonio inmueble) se mantengan en activo. Por tanto, la conservación de las salinas supone también una garantía de continuidad para un oficio, el de los maestros salineros, que guarda una importante carga histórica ligada a los inicios de la actividad, los conocimientos acumulados y transmitidos durante varias generaciones por parte de los maestros salineros canarios como parte inseparable de este legado histórico. Las salinas representan también un modelo de interacción ambiental entre el aprovechamiento de los recursos y las condiciones del medio natural. De esta manera se comportan como marisma artificial generando un ecosistema singular en el que tienen su hábitat un número importantes de especies naturales y vegetales que incorporan valores añadidos a los reseñados, especialmente con la fauna, como estación de paso y avituallamiento de las aves migratorias. (4)

Los paisajes culturales aparecen como refugios de la identidad, como singularidades de gran belleza y significado histórico, como espacios con memoria con los que la sociedad busca recuperar la relación con un territorio cada vez más homogéneo y aterritorial. (5)

El paisaje es una realidad física que integra valores tan diversos como son los ambientales, culturales, históricos y estéticos. Para las personas existen básicamente dos tipos de paisajes, de un lado están los paisajes naturales que son aquellos en los que sus elementos tales como el relieve, la vegetación, el agua y el suelo están poco trasformados por el ser humano y de otro lado, están los paisajes culturales, que son el resultado de las transformaciones de los paisajes naturales por parte de la humanidad a través de la historia.

En numerosas ocasiones el paisaje no siempre es tomado como un recurso en sí mismo porque su estudio se realiza de forma pormenorizada desde diversas ópticas relativas a los estudios del paisaje territorial, del paisaje rural, del paisaje urbano, del paisaje social, del paisaje cultural, etc. Los valores del paisaje se consideran fundamentales para desarrollar propuestas de ordenación territorial así como ecológicas y culturales. Las estrategias clásicas de conservación de los diversos espacios salineros suelen ser de dos tipos, por un lado, se apuesta por mantener la actividad en las salinas, cada vez más en declive, aunque no se rijan por la productividad de éstas y, por otro lado, mediante la protección por la legislación según indica la Ley de Patrimonio Histórico Español.

Sin embargo, se ha constatado que, aunque se hayan incoado numerosos expedientes para proteger legislativamente a las salinas, existen varios expedientes de incoación que han caducado, por lo que se encuentran en la actualidad en un vacío jurídico importante que favorece la erosión de los diferentes elementos de estos espacios singulares, al carecer de uso y de mantenimiento de las instalaciones situadas en determinados entornos marítimo-terrestres de las islas.

En el preámbulo de la Carta de Cracovia de 2000 indica "Cada comunidad, teniendo en cuenta su memoria colectiva y conscientes de su pasado, es responsable de la identificación, así como de la gestión de su patrimonio. Los elementos individuales de este patrimonio son portadores de muchos valores, los cuales pueden cambiar en el tiempo. (...) Este patrimonio no puede ser definido de un modo unívoco y estable. Sólo se puede indicar la dirección en la cual puede ser identificado”. (6)

Desde el punto de vista del paisajismo, existe una experiencia singular de intervención consistente en generar un paisaje híbrido de naturaleza y cultura creado según las directrices de un proyecto denominado "La Tancada" en los campos de sal del Parque Natural del Ebro, en Tarragona. 
El Proyecto que recibió el Premio de intervención urbana en el espacio exterior y el Premio de Turismo Responsable, ambos concedidos por la Comunidad Autónoma de Cataluña en 2013, se ejecutó en el año 2012 y se sitúa en el extremo sur del Río Ebro Delta, uno de los ríos más importantes y caudalosos de la Península Ibérica.

La intervención consistió en la construcción de un paisaje a modo de un ecosistema artificial (figura 1) que propone un conjunto de tramos geométricos para ser recorridos por los visitantes (figura 2).

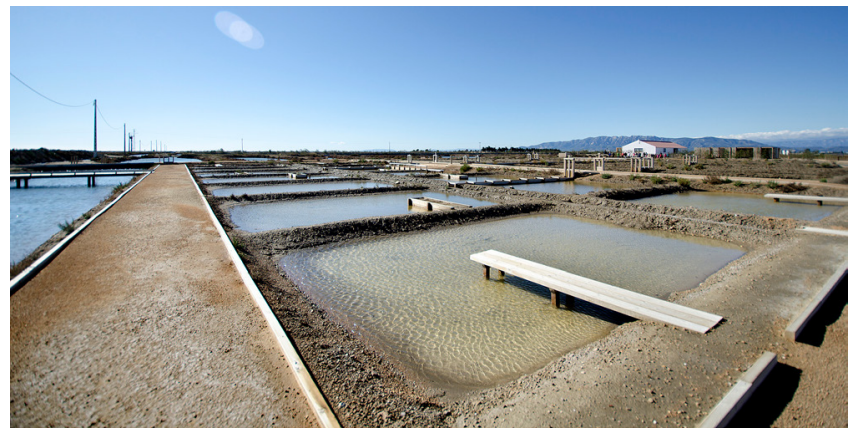

Figura 1. Paisaje híbrido "La Tancada" en el Parque Natural del Ebro, Tarragona. España.

Fuente. Sergi Romero.

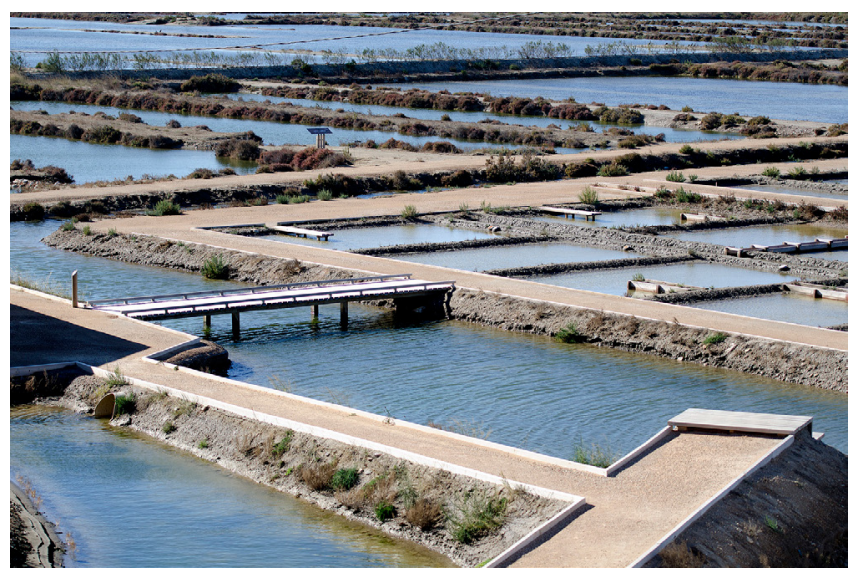

Figura 2. Paisaje híbrido "La Tancada" en el Parque Natural del Ebro, Tarragona. España.

Fuente. Sergi Romero.

En el año 2009 se produjo un acuerdo entre la Fundación Natural Catalunya Caixa, el Ministerio de Medio Ambiente de España, el Parque Natural Delte de l'Ebre y el Instituto de investigación Agrícola lograron junto con la Unión Europea el denominado programa LIFE que tenía como finalidad restaurar este sitio y otras áreas naturales cercanas. Del proyecto LIFE surge otro proyecto denominado "MON NATURA" que consistió en crear un Centro de Interpretación para convertir la explanada existente en un paisaje líquido y salado que realiza una magnífica interacción con el ciclo de luz. El diseño del proyecto construye un híbrido de naturaleza y cultura que dignifican las peculiaridades del sitio, tanto en términos de los ecosistemas artificiales para las especies en peligro de extinción como en la construcción de este paisaje natural-artificial que propone formas alternativas y sugerentes en el sitio para los visitantes, dentro de un contexto que los invita a explorar este peculiar paisaje: Lagunas intermareales, campos de sales y cristalizaciones, así como la presencia de flamencos, charranes, etc. (7)

En la comunidad autónoma de Andalucía existe una experiencia plausible consistente en el cambio de uso de una salina tradicional a una nueva actividad, la acuicultura. Las infraestructuras salineras de Astur (figura 3) están situadas en el término municipal de Punta Umbría en Huelva dentro del Paraje Natural Marismas del Odiel y que constituye uno de los ecosistemas más productivos del litoral Sur-atlántico en el que destaca una rica ictiofauna y una variada avifauna.

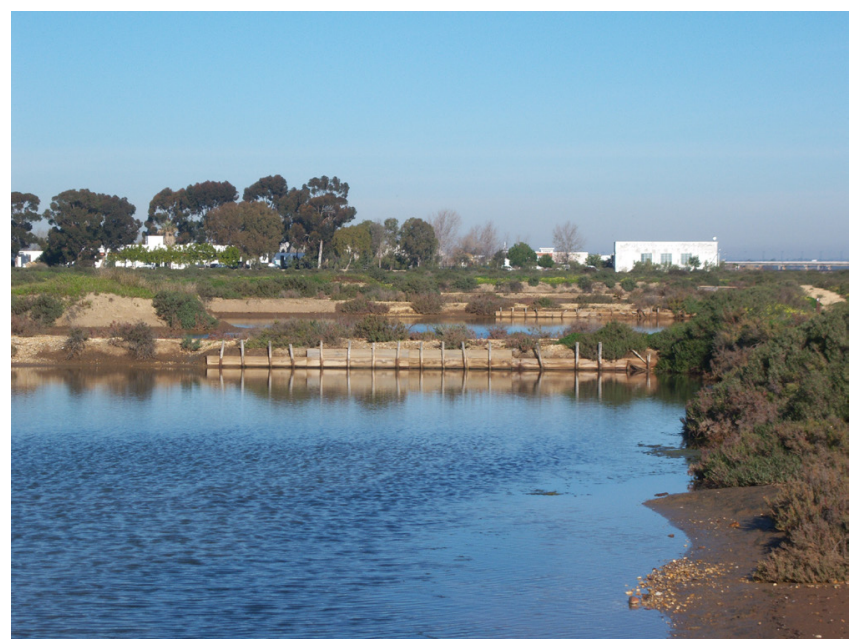

Figura 3. Salinas de Astur. Paraje Natural de las Marismas de Odiel. Punta Umbria. España.

Fuente. http://www.juntadeandalucia.es/medioambiente/servtc5/ ventana/img/fotos/SENDEROS/PjN_MARISMAS_DEL_ODIEL/ SALINAS_BACUTA/1083_SS_05.jpg

Las salinas del Astur se construyeron en la década de los veinte del pasado siglo XX. Originariamente se desecaron y se sanearon las marismas para dedicarlas al cultivo y ganadería, sin embargo, por ser un terreno pobre para la agricultura y siendo la sal el principal conservante alimenticio de aquellos años, se transformó su uso en salina, que estuvo en funcionamiento hasta la década de los setenta del siglo XX, cuando fue abandonada por falta de rentabilidad. En el año 2001 se inició la actividad de la acuicultura en tres de las antiguas naves o esteros de la salina, con el objetivo de producir doradas, lubinas y lenguados. (8)

El sistema de las salinas de Astur funciona como un estuario donde los cauces los ríos Tinto y Odiel depositan al desembocar gran cantidad de sedimentos cargados de nutrientes. Éstos son la principal base alimenticia de las aves que pueblan este espacio natural. A su vez, este aporte continuo de sedimentos y la fuerza de las mareas generan islas separadas por extensos brazos de agua como la Isla de Enmedio, la de Saltés y la de Bacuta localizadas al Sur del enclave. Parte de su riqueza ecológica estriba en la variedad de paisajes que conforman este enclave. Al visitante le llama la atención su gran horizontalidad que le permite disfrutar del olor del salitre, de la brisa marina y de la espectacular llegada de centenares de aves que encuentran en esta marisma un lugar idóneo donde nutrirse y poder nidificar. (9)

Desde el punto de vista del paisaje urbano, existe una intervención muy significativa que se llevó a cabo en la isla de 
Lanzarote y concretamente en las salinas de Matagorda, en el municipio de Tías donde se propuso un cambio de uso. Los autores del proyecto de rehabilitación apostaron por un concepto de reutilización de las salinas introduciendo un concepto diferente de parque urbano o de zona verde. La intervención consistió en crear un parque de zonas húmedas a base de láminas de agua de uso público (figura 4) a partir de una forma preexistente en el territorio.

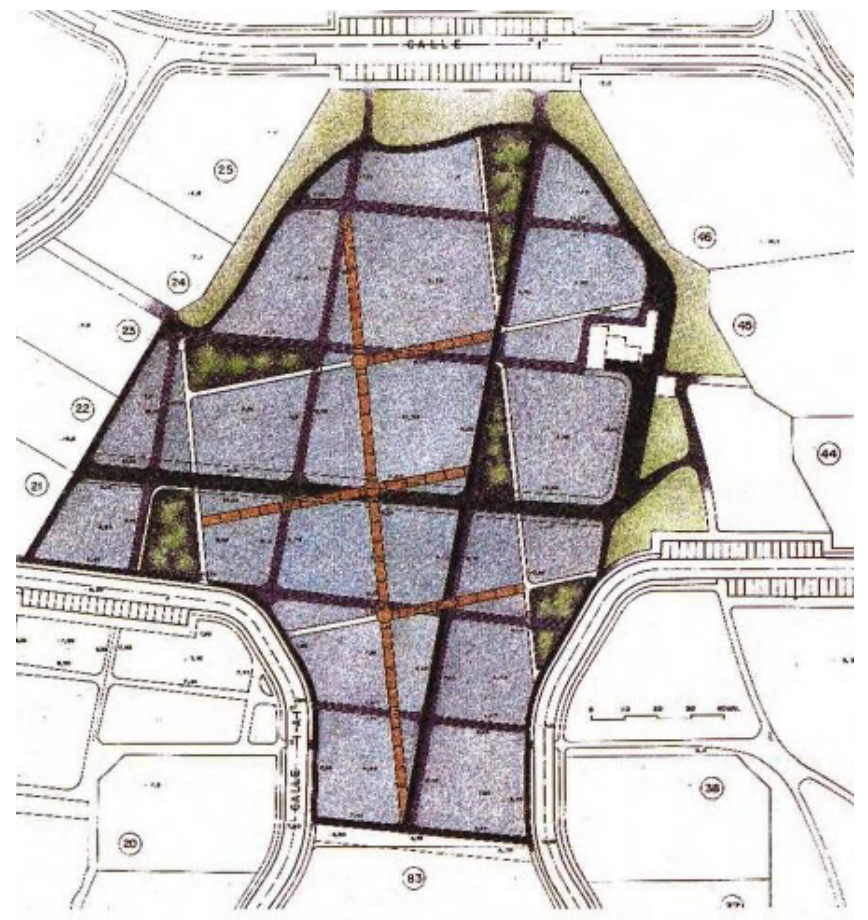

Figura 4. Planta general de la intervención en las salinas de Matagorda. Municipio de Tías. Lanzarote. España.

Fuente. https://www.coam.org/media/Default\%2oFiles/fundacion/biblioteca/revista-urbanismo/docs/revista-urbanismo-n4-pag97-10o.pdf

La utilización y el disfrute de las láminas de agua formalmente organizadas junto con la realización de varios itinerarios peatonales fueron los puntos de partida de esta propuesta de intervención. Para conseguirlo, suministran agua de mar a los cocederos mediante la reutilización del molino de viento salinero que bombea agua a los niveles superiores de las salinas con la finalidad de crear un recorrido acuático que permita el paso del agua de unos cocederos a otros por gravedad, evitando así el estancamiento del agua y, por consiguiente, la proliferación de mosquitos y otros insectos. (10)

Sin embargo, con el paso del tiempo y debido al escaso mantenimiento de las instalaciones, al excesivo gasto energético de las bombas de impulsión necesarias para renovar las láminas de agua, así como la presencia continua de mosquitos en la zona se optó por sustituir el agua por una capa de árido volcánico a modo de un jardín seco (figura 5).

Algunos de los entornos urbanos que se han creado en las Islas Canarias tienen su origen en los terrenos donde se asentaban las infraestructuras salineras tradicionales y por lo tanto, han mostrado un escaso interés hacia los recursos paisajísticos existentes. Los territorios salineros suelen ser enclaves de carácter singular donde confluyen intereses contrapuestos como son los económicos, sociales, ecológicos y culturales. La

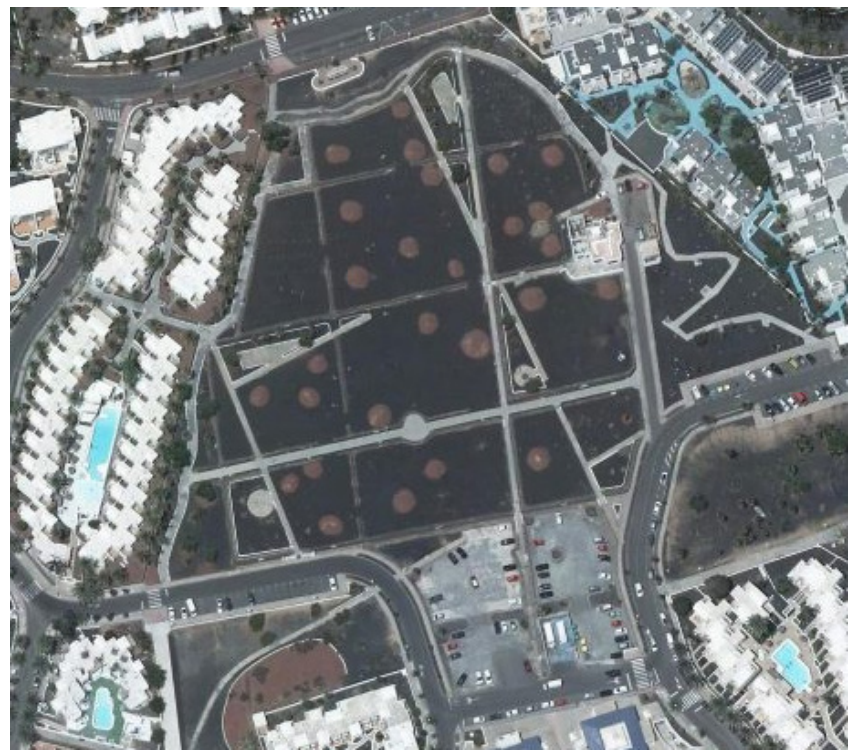

Figura 5. Intervención en las salinas de Matagorda. Municipio de Tías. Lanzarote. España.

Fuente. IDECanarias Visor 4.5.1

presión urbanística y la decadencia de la industria salinera tradicional han permitido que los espacios salineros fuesen ocupados por urbanizaciones que han terminado por desnaturalizar al paisaje del litoral marítimo-terrestre de las islas.

Los riesgos ocasionados por el abandono de la actividad agrícola a favor de los espacios urbanos, el turismo y sus servidumbres, desequilibran factores esenciales del paisaje así como la pérdida de los usos tradicionales. En este sentido, pueden servir como ejemplos algunas de las salinas de la isla de Lanzarote como lo son las de Puerto Naos en Arrecife, las de Puerto del Carmen y las de Matagorda en Tías.

Desde un punto de vista cultural y turístico, cabe reseñar la intervención que se llevó a cabo en las salinas de los Cancajos en el municipio de Breña Baja en la isla de La Palma. Los trabajos consistieron en rehabilitar los tajos grandes y los

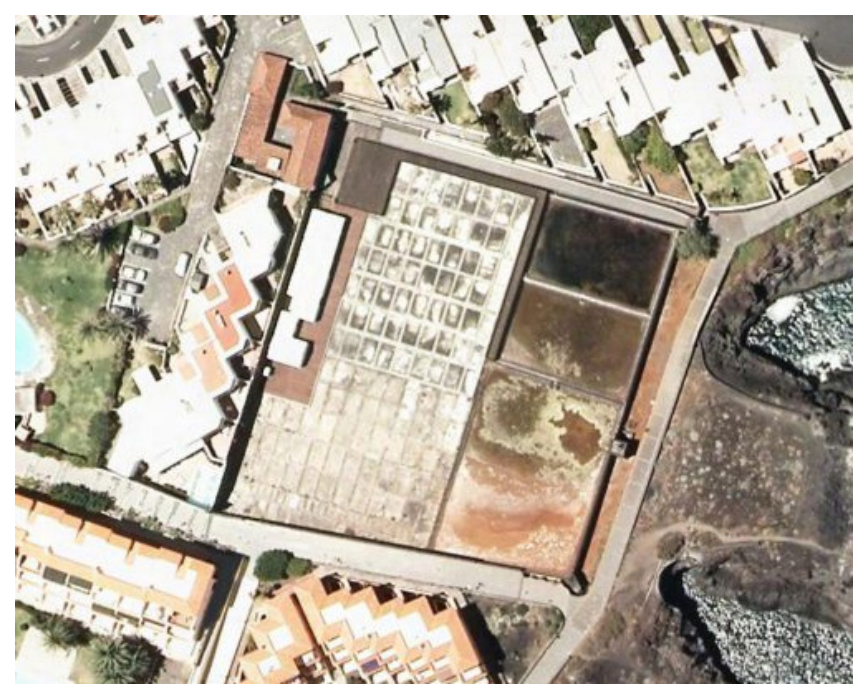

Figura 6. Salinas de Los Cancajos. Municipio Breña Baja. La Palma. España.

Fuente. IDECanarias Visor 4.5.1 
tajos pequeños, los cocederos, el sistema de impulsión de agua de mar mediante bombas que funcionan con energía eléctrica y algunas actuaciones puntuales en el edificio existente vinculado a las salinas para convertirlo en Centro de Interpretación de la sal. Las salinas de los Cancajos actualmente están encorsetas en una manzana del tejido urbano de uso turístico (figura 6). Los tajos de dicha infraestructura se construyeron en su inicio de una forma diferente a como se realizaba en las salinas de las islas orientales del archipiélago canario como lo son en Gran Canaria, Fuerteventura y Lanzarote.

En el caso concreto de los Cancajos, los tajos están construidos con mortero de cal y por lo tanto, es dificultosa la puesta en funcionamiento de la instalación para la extracción de sal ya que el agua de mar se elimina por evaporación y no se filtra hacia el fondo de los tajos tal y como sucede con las salinas con fondo de barro construidas en las islas orientales.

Con el paso del tiempo y aun siendo rehabilitadas en el año 2004, actualmente dicha infraestructura salinera presenta síntomas importantes de deterioro ante la falta de uso y del mantenimiento necesario para garantizar su conservación (figura 7). Las instalaciones se encuentran cerradas al público en general y, por consiguiente, no se ha conseguido con la intervención llevada a cabo regenerar este espacio salinero como se pretendía para que fuese un polo de atracción cultural de un entorno urbano de por sí de uso turístico.

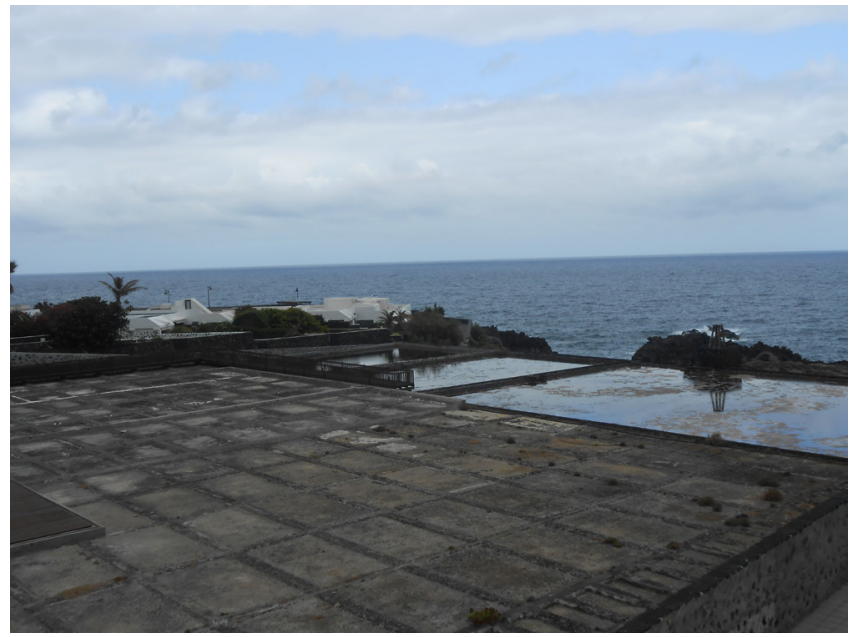

Figura 7. Salinas de Los Cancajos. Municipio Breña Baja. La Palma. España.

Fuente. Víctor Manuel Cabrera García

Ante la inevitable degradación que presentan las salinas de las Islas Canarias debido a la escasez de los recursos económicos necesarios para garantizar su conservación y debido a la falta de actividad en las mismas, se plantea la necesidad de realizar un estudio de cómo podríamos recuperar dicho $\mathrm{Pa}$ trimonio Cultural con la finalidad de devolverles algunos de los valores que han perdido con el paso del tiempo para que puedan convertirse en un reclamo, tanto para los lugareños como para los visitantes.

\section{MATERIALES Y MÉTODO}

La presente investigación se lleva a cabo de acuerdo a las siguientes pautas:
- Determinación de objetivos

- Enfoque metodológico

- Análisis de resultados

\section{Determinación de objetivos}

El objetivo principal es conocer la situación actual que presentan las salinas de las Islas Canarias y plantear estrategias de intervención desde la óptica del paisaje con la finalidad de revitalizar los espacios salineros que actualmente se encuentran mayoritariamente en desuso y abandonados.

Entre los objetivos específicos se establecen los siguientes:

- Identificar las razones del deterioro y abandono.

- Proponer posibles estrategias de intervención con la finalidad de revitalizar a las infraestructuras salineras provenientes de la cultura industrial tradicional.

\section{El enfoque metodológico}

Se divide en dos fases:

\section{- Fase exploratoria}

En una primera fase, se ha obtenido información de diversas fuentes provenientes de estudios realizados acerca de la problemática de las salinas de Canarias, tales como legislaciones, libros, anuarios económicos del sector de la sal, bases de datos de las administraciones, publicaciones académicas, jornadas temáticas, etc.

\section{- Análisis general}

Inicialmente se realiza un análisis de la situación y se ha obtenido una visión completa de la situación en referencia a:

a. El sector de la sal y los problemas que ha tenido a lo largo del tiempo.

b. El estado de las instalaciones y sus elementos.

La investigación atiende fundamentalmente a las relaciones que emanan de la realidad existente, tratando de encontrar las razones por las que se ha producido el deterioro de las salinas y analizar las diversas experiencias existentes en la recuperación de estos espacios marítimos -terrestres.

\section{Análisis de resultados}

Se ha llevado a cabo mediante el estudio de casos referidos a los posibles cambios de usos que podrían albergar las salinas que están abandonadas y deterioradas con la finalidad de evitar en la medida de lo posible la desaparición de estas infraestructuras procedentes de la cultura industrial tradicional.

\section{DISCUSIÓN Y RESULTADOS}

La salina artesanal colabora de forma peculiar con el medioambiente permitiendo el desarrollo de ecosistemas que de otro modo no tendrían lugar. Además, debido a su producción no muy elevada, no cuenta con facilidad para competir en el mercado y esto ha provocado el abandono de muchas de ellas. Otras han sabido reinventarse, consiguiendo sostenerse económicamente gracias a la innovación en los productos relacionados con la sal, e introduciendo actividades culturales y 
relacionadas con la salud en las propias salinas. Es importante destacar que la salina artesanal contribuye al patrimonio nacional. Su producción favorece el desarrollo de paisajes y ecosistemas que de otro modo no serían posibles. Es necesario poner en valor su contribución al medio ambiente, sobre todo cuando la forma de extracción es completamente artesanal, pues en este caso genera impacto sobre el medio modificando el paisaje, pero aportando un gran valor y favoreciendo la biodiversidad. (11)

Existen muy pocas salinas tradicionales donde se trabaje a mano destacan Cádiz (Salina La Esperanza, El Águila), Huelva, Álava (Salinas de Añana) y Canarias (salinas de Fuencaliente y Tenefé) si bien los productos comercializados no suponen un porcentaje ni siquiera residual en el mercado. (12)

Las salinas artesanales del archipiélago canario tienen un volumen de extracción de sal marina muy inferior a las salinas industriales y los datos son marginales respecto a otras comunidades autónomas. Según el informe redactado por el Estudio Luengo S.L. sobre las salinas de Canarias, la producción de sal en el conjunto de las salinas genera un volumen de escasamente las 3000 toneladas/año. Gran parte de ese volumen se tiene que desechar debido al efecto del polvo por el deterioro del entorno circundante de las salinas. En este sentido, el volumen que se extrae representa un $6 \%$ de la sal que se consume en el mercado local y algunas de las salinas se mantienen anegadas para impedir su deterioro como son las salinas de la Florida y las salinas de Tenefé, ambas ubicadas en la isla de Gran Canaria. Desde que finalizó la actuación del Programa Regis de recuperación de las salinas de Canarias en el año 1997, que no sólo iba dirigido a rehabilitar las salinas sino que se pretendía intervenir en el mercado de la sal. Si bien las salinas ocupan un suelo de alto valor económico, su posible rehabilitación y mantenimiento suponen un elevado coste económico que no se corresponde con el beneficio de la venta de la sal en el mercado. (13)

En la actualidad algunas de las salinas del archipiélago canario se encuentran protegidas a nivel urbanístico y legislativo, aunque dicha protección parece tener un carácter meramente simbólico ya que dichas infraestructuras sufren un grave proceso de deterioro ante las tareas inexistentes de conservación. Las salinas artesanales existentes en las islas forman parte del Patrimonio Histórico Canario ya que contribuyen a la sostenibilidad de una parte concreta del territorio insular y además, reconoce el valor patrimonial y cultural que tienen para la ciudadanía. Por lo tanto, es necesario mantener el entorno ambiental y paisajístico más allá del estricto espacio físico que ocupan dichas infraestructuras.

Las Islas Canarias poseen un extenso número de infraestructuras salineras que han quedado sin uso debido a los diversos cambios sociales y de los modelos económicos. Conservar los espacios con valores patrimoniales por su paisaje natural y/o artificial no supone prohibir el desarrollo de nuevas actividades ya que recuperar y proteger sin usos concretos resulta ser una acción en vano. En este sentido ¿Existen alternativas distintas a las ya ensayadas para recuperar, incorporar y revitalizar las salinas de las Islas Canarias para la sociedad actual?

Las intervenciones de cambio de uso constituyen una estrategia contrastada para conseguir el mantenimiento y la salvaguarda de este patrimonio cada vez más extenso. El carácter de dichas intervenciones es muy diverso, dependiendo de cada situación y de la consideración concreta de cada edificio como bien patrimonial, la adopción de las decisiones fundamentales referentes al nuevo uso, los elementos o visiones espaciales a conservar, las técnicas constructivas a aplicar o los elementos susceptibles de supresión. Su catalogación y grado de protección constituyen, evidentemente, el marco inicial de referencia para pautar el proyecto de intervención. (14)

Actualmente el sector turístico representa la principal fuente de ingresos de la Comunidad Autónoma de Canarias y las tendencias de la demanda turística suelen ser la singularidad, la diferenciación y la autenticidad. La apuesta por un turismo sostenible enfocado hacia el Patrimonio, la Cultura y su Paisaje supondría una contribución eficaz al desarrollo económico de cualquier territorio y es por ello por lo que un paisaje atractivo es un factor clave para el desarrollo económico de sus localidades y de sus gentes.

Las salinas como paisaje cartesiano, abstracto y funcional tienen hondas resonancias en nuestra cultura. El paisaje de la salina, la perspectiva visual de las montañas de sal sobre la trama ortogonal adquiere dimensiones surrealistas. El sistema de producción en las salinas nos recuerda el orden de las plantaciones vegetales en sus alineamientos, de la misma manera que el cuidado y la limpieza de sus labores lo acerca a la jardinería, en su tradición más clásica. (15)

Como propuesta alternativa de intervención paisajística a realizar en las salinas que han quedado en desuso, se propone crear unos jardines luminiscentes basados en el arte vitral y en el fenómeno natural de la bioluminiscencia que se observa en algunos organismos marinos (plancton, fitoplancton, etc.).

La biolumiscencia es la propiedad que tienen algunos seres vivos de emitir luz (16). Se trata de un proceso que permite a determinados organismos vivos producir una luz fría, resultado de una reacción bioquímica ante determinadas situaciones para asegurar la supervivencia. La intervención que se propone permitiría contemplar un tipo de paisaje nocturno a través de la emisión de luz a través de la trama geométrica de las salinas. Estos nuevos espacios libres podrían convertirse en nuevos polos de atracción para el turismo cultural, lo que posibilitaría crear nuevas experiencias de carácter sensorial que aportan nuevos valores añadidos respecto a las diversas rutas culturales y patrimoniales de unos paisajes marinos tan singulares y domesticados por la humanidad en la obtención de la sal.

El fenómeno de la bioluminiscencia se puede observar en las noches oscuras y fundamentalmente en los climas cálidos como es el caso de las playas de las islas Maldivas (figura 8), Puerto Rico, México, California, etc. El agua del mar emite una misteriosa luz azul cuando las olas rompen en la arena de las playas dejando un paisaje iluminado de carácter embriagador de difícil descripción denominado "mar de estrellas".

Los jardines luminiscentes en horario diurno estarán basados en los vitrales, que se consideran como una manifestación artística que emplea vidrios de colores junto con otros elementos como la luz natural, la atmósfera, el fondo, la forma, la textura, el movimiento, etc. donde el vitral se muestra vivo debido a la intensidad variable de la luz solar a lo largo del día. Sin embargo, en la oscuridad de la noche, se utilizará la propiedad que tienen algunos cuerpos de emitir luz fría (bioluminiscencia) sin elevar la temperatura. Se tendrán 


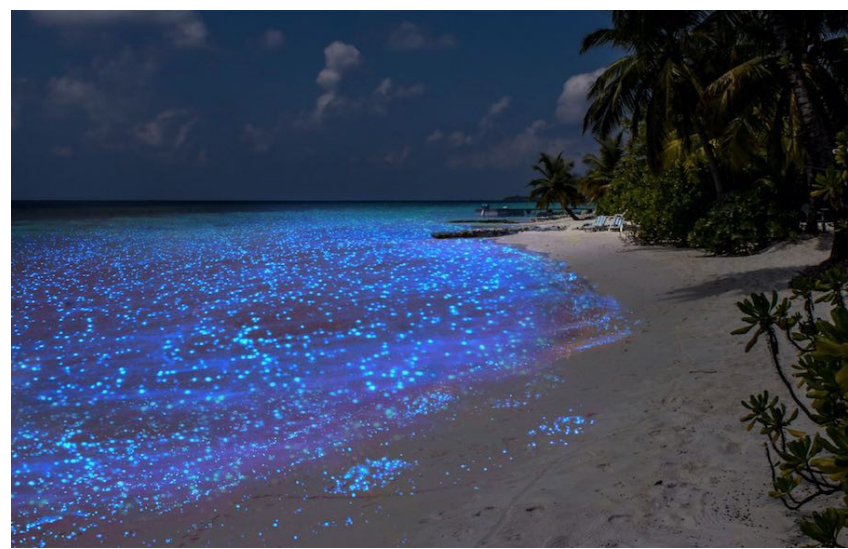

Figura 8. La bioluminiscencia en la Playa de Vaadhoo. Islas Maldivas.

Fuente. https://www.nauticalnewstoday.com/mejores-playas-del-mundo-mar-de-estrellas/

que emplear cuerpos sólidos donde sus estructuras atómicas absorban la energía procedente de la radicación solar y posteriormente la desprenda cuando los electrones vuelven a su estado original.

Para conseguir el efecto de vitral nocturno se apuesta por la utilización de materiales fotoluminiscentes capaces de absorber la radiación ultravioleta procedente de la luz del sol para posteriormente emitir luz fría en la oscuridad de la noche durante horas. Como método de ejecución se emplearán áridos fotoluminiscentes enriquecidos con aluminato de estroncio que pueden ser integrados en el pavimento (figura 9) o como elementos decorativos sueltos depositados sobre los tajos y los cocederos de las salinas.

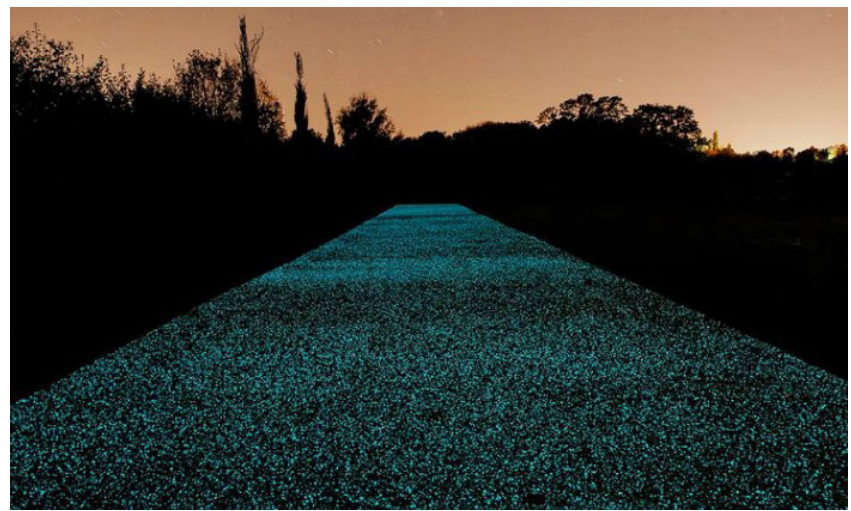

Figura 9. Áridos enriquecidos con aluminato de estroncio incorporados al pavimento.

Fuente. https://luminiscentecanarias.es/piedrasluminiscentes/

Los jardines luminiscentes evocarán a los vitrales de colores donde el dinamismo inherente y cambiante de la luz permiten al espectador observar una alteración de la intensidad de los colores. Los visitantes podrán percibir nuevos estímulos sensoriales en la contemplación de la trama geométrica abstracta de los espacios salineros debido a que los materiales fotoluminiscentes que se emplearán en los tajos y en los cocederos se observarán como alfombras o tapices de colores inspirados en los tonos de colores que deja un alga microscópica que vive en las salinas denominada "Dunaliella salina" (figura 10).

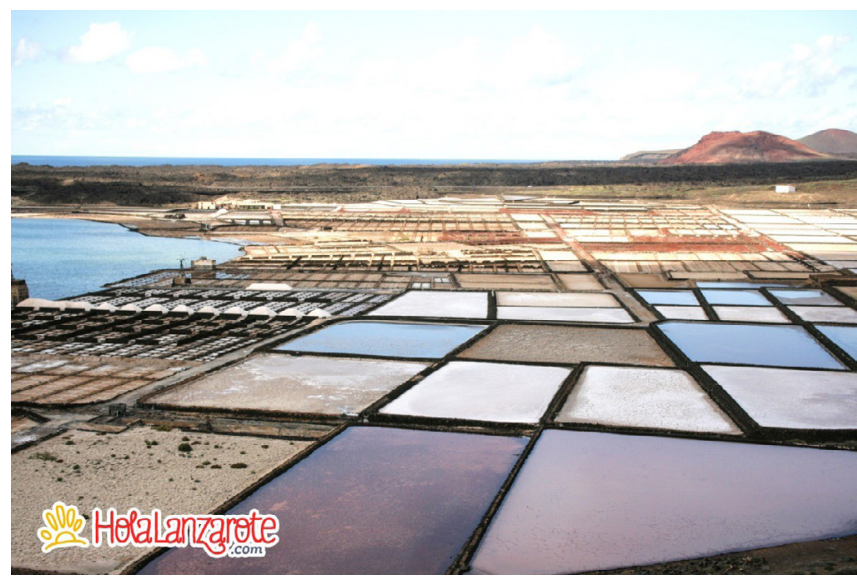

Figura 10. Salinas del Janubio. Municipio de Yaiza. Lanzarote. España.

Fuente. https://holalanzarote.com/images/big/salinas-del-janubio-21.jpg

El paisaje nocturno puede manifestarse como una escenografía en muchos lugares idealizada y de colores. La luz como fuente generadora de colores, es un complemento, que además de guiar en el caminar, nos provoca sensaciones. (17)

Para garantizar unos niveles de iluminación adecuados en horario nocturno para el tránsito de las personas a través de los jardines luminiscentes en los diversos espacios salineros también es necesario disponer de un alumbrado público complementario. En este sentido se optaría por utilizar luminarias fotovoltaicas que funcionan con energía renovable solar, lo que aporta un alto valor añadido de eficiencia energética y que se consigue además con el empleo de las lámparas LED. Adoptando sistemas de iluminación inteligentes así como el empleo de las energías renovables se obtendrían múltiples beneficios a un coste muy reducido, ahorraríamos energía y evitaríamos los efectos perniciosos de la contaminación lumínica. No se trata de apagar todas las luces, sino más bien de saber que debemos y como debemos de iluminar.

El cielo del archipiélago canario está protegido por la Ley del Cielo del 31 de octubre de 1988 La normativa regula cuatro aspectos fundamentales: la contaminación lumínica, la contaminación radioeléctrica, la contaminación atmosférica y las rutas aéreas (18). La entrada en vigor de dicha Ley ha condicionado a las instalaciones de alumbrado público estableciendo límites máximos referidos al flujo luminoso nominal de las lámparas destinadas al alumbrado público en los viales, los edificios y los monumentos, los jardines, las playas, los parques, las áreas naturales, los letreros, etc. Si se diseñara el alumbrado público compatible con el medioambiente no solo fomentamos el ahorro energético y no favorecemos el cambio climático, sino que también respetamos la biodiversidad y el cielo nocturno.

Los efectos de la contaminación lumínica van mucho más allá de la pérdida creciente de un patrimonio común: el derecho a observar las estrellas. Algunas reservas de la biosfera españolas son pioneras a nivel mundial a la hora de dar respuestas a este fenómeno, ya sea desde la óptica de la eficiencia energética, de la recuperación de la calidad del cielo o desde el mundo de la conservación de la biodiversidad. Además, fue precisamente en la Reserva de la Biosfera de la isla de La Palma donde surgió en 2007 la Iniciativa Starlight (La Luz de las Estrellas), 
asociada al Programa MaB de la UNESCO que hoy representa el primer movimiento mundial en favor de la calidad del cielo nocturno y la limitación de la contaminación lumínica.

La Declaración Starlight propugna en su primer punto que "el derecho a un cielo nocturno no contaminado que permita disfrutar de la contemplación del firmamento debe considerarse como un derecho inalienable de la Humanidad, equiparable al resto de los derechos ambientales, sociales y culturales, atendiendo a su incidencia en el desarrollo de todos los pueblos y a su repercusión en la conservación de la diversidad biológica”. En el artículo 7 se especifica la necesidad de limitar los efectos adversos de la iluminación artificial al establecer que "ha de promoverse el uso racional de la iluminación artificial, de tal forma que el resplandor que provoca en el cielo se reduzca a un mínimo aceptable, evitando igualmente los impactos nocivos sobre los seres humanos y la vida en la naturaleza. Las administraciones públicas, la industria de la iluminación y los principales actores que inciden en la toma de decisiones, han de asegurar un uso responsable de la luz artificial por parte de todos los usuarios, integrando esta dimensión en la planificación y en las políticas de sostenibilidad energética, las cuales habrán de apoyarse en mediciones de la contaminación lumínica, tanto desde la tierra como desde el espacio. Tal actitud implica un uso más eficiente de la energía en consonancia con los acuerdos sobre el cambio climático y la protección del medio ambiente". (19)

\section{CONCLUSION}

Recuperar el patrimonio salinero canario que se encuentra actualmente en desuso y abandonado mediante la incorporación de nuevos usos que sean compatibles con las salinas supone un reto importante para la nuestra sociedad. En este sentido surgen incertidumbres respecto a los tipos de intervención más adecuadas que se pueden realizar si atendemos a las directrices de conservación que se especifican en las legislaciones patrimoniales.
La catalogación, el estudio y la protección del patrimonio salinero tan solo son instrumentos que garantizan una cierta conservación de este para transmitirlo a las generaciones futuras. La escasez de recursos económicos destinados a las tareas de mantenimiento del extenso patrimonio salinero que atesora la Comunidad Autónoma de Canarias en aras de garantizar su conservación favorece la aparición de la denominada ruina funcional en los diferentes elementos constructivos de las salinas y, por consiguiente, se facilita la pérdida de una parte sustancial de la memoria histórica de las sociedades que las construyeron. Es imprescindible por lo tanto convertir al patrimonio salinero en un recurso activo ya que, abandonado, inactivo o en ruinas no sirve de nada.

Las salinas son espacios abiertos y accesibles que son proclives a tener actos vandálicos o de robos de sus elementos si no se encuentran en explotación. Otras veces, la presión urbanística e industrial amenazan el entorno de estas infraestructuras territoriales, que con frecuencia llegan hasta los propios límites de estas y se ven afectadas incluso por los vertidos de basura de naturaleza variable. Estos condicionantes, entre otros, suponen un riesgo para su conservación, por lo tanto, es necesario crear programas de sensibilización de la población.

Las instalaciones territoriales salineras constituyen un atractivo cultural, medioambiental y paisajístico de primera magnitud. Se pretende crear nuevas experiencias sensoriales que aporten un valor añadido respecto a las diversas rutas culturales y patrimoniales posibilitando recuperar y conservar lo que aún no se perdido de estas infraestructuras procedentes de la cultura industrial tradicional.

"Respetar lo pasado renovando la tradición, es una de las maneras más hondas de fraguar porvenir y hacer progreso".

Miguel de Unamuno

\section{REFERENCIAS}

(1) Román López, Emilia (2014) “El patrimonio territorial como base para un nuevo modelo de desarrollo territorial. Las salinas en el territorio: paisaje y patrimonio". VII Congreso Internacional de Ordenación del Territorio, Madrid.

(2) Convenio Europeo del Paisaje, Florencia 200o. https://rm.coe.int/CoERMPublicCommonSearchServices/DisplayDCTMContent?documentId=09000016802f3fbd [Consulta 27.01.2020]

(3) España. Ley 4/1999, de 15 de marzo, de Patrimonio Histórico de Canarias, modificada por la Ley 11/2002, de 21 de noviembre. Gobierno de Canarias.

(4) Cabrera García, Víctor Manuel (2019) “El paisaje salinero canario. Alternativas para su revitalización”. GE-CONSERVACIÓN, Vol. 1, No 15, pp 50-61. https://doi.org/10.37558/gec.v15io.599

(5) Mateu i Lladó, Jaime (2014) "los paisajes culturales patrimonio mundial como herramientas de gestión territorial. El caso de la serra de tramuntana de mallorca”. Boletín de la Asociación de Geógrafos No 66, pp. 253-270. https://doi. org/10.21138/bage.1789

(6) Carta de Cracovia (2000) "Principios para la conservación y restauración del patrimonio construido". Astrágalo: revista cuatrimestral iberoamericana, $\mathrm{N}^{0} 17,2001$, pp. 127-134.

(7) https://www.plataformaarquitectura.cl/cl/o2-365898/arquitectura-y-paisaje-campos-de-sal-la-tancada-convertidos-en-un-paisaje-liquido-por-emf [Consulta 10.01.2020]

(8) https://www.salinasdelastur.com/ [Consulta 20.02.2020]

(9) http://www.juntadeandalucia.es/medioambiente/servtc5/ventana/mostrarFicha.do;jsessionid=1F422147A9789F1510BF925B6oD9EC28?idEspacio=7393 [Consulta 16.03.2020]

(10) https://www.coam.org/media/Default\%20Files/fundacion/biblioteca/revista-urbanismo/docs/revista-urbanismo-n4-pag97-100.pdf [Consulta 30.03.2020]

(11) González Halcón, Carmen (2018) "Estudio de viabilidad de producción anual de la salina en marisma con energías renovables". Universidad Politécnica Comillas. Madrid.

(12) Mier-Terán, Juan José (2018) "Estudio de mercado de la Sal en España”. Comercialización e investigación de Mercados. Universidad de Cádiz 
(13) Luengo Barreto, Alberto; Marín Cabrera, Cipriano (2008) "Informe Salinas Canarias”. Tomo 1. Estado actual de las salinas canarias. Pasado y presente. Estrategias de actuación.

(14) Cornado Bardón, Cossima (2008) "Nuevos usos para el patrimonio difuso". Comunicación de congreso, pp. 7-12.

(15) Pasado, presente y futuro de las Salinas Canarias. Jornadas de fomento de las salinas tradicionales de Canarias organizadas por la Conserjería de Agricultura, Ganadería, Pesca y Alimentación. Viceconsejería de Ordenación del Territorio y por la Asociación de Productores de Sal Marina de Canarias. Septiembre 2010.

(16) Real Academia Española de la lengua R.A.E (2019).

(17) Prat Mendoza, Francisca (2009) “Paisaje nocturno \& contaminación lumínica”. Universidad Central de Chile.

(18) España. Ley 31/1988, de 31 de octubre, sobre Protección de la Calidad Astronómica de los Observatorios del Instituto de Astrofísica de Canarias.

(19) Declaración sobre la defensa del cielo nocturno y el derecho a la luz de las estrellas (2007). STARLIGHT Patrimonio de todos. Conferencia Internacional en Defensa de la Calidad del Cielo Nocturno y el Derecho a Observar las Estrellas. Declaración de La Palma. 